NASA/CR-97-206269

ICASE Report No. 97-69

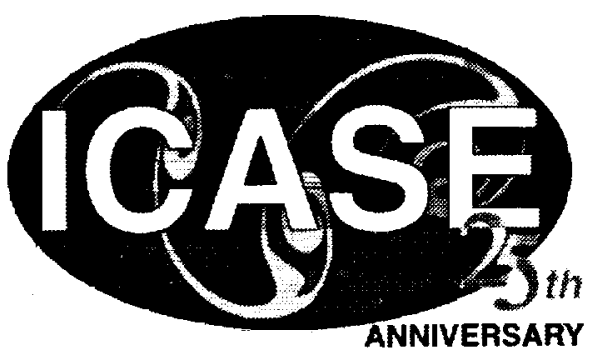

\title{
Admitting the Inadmissible: Adjoint Formulation for Incomplete Cost Functionals in Aerodynamic Optimization
}

Eyal Arian and Manuel D. Salas 


\section{The NASA STI Program Office ... in Profile}

Since its founding, NASA has been dedicated to the advancement of aeronautics and space science. The NASA Scientific and Technical Information (STT) Program Office plays a key part in helping NASA maintain this important role.

The NASA STI Program Office is operated by Langley Research Center, the lead center for NASA's scientific and technical information. The NASA STI Program Office provides access to the NASA STI Database, the largest collection of aeronautical and space science STI in the world. The Program Office is also NASA's institutional mechanism for disseminating the results of its research and development activities. These results are published by NASA in the NASA STI Report Series, which includes the following report types:

- TECHNICAL PUBLICATION. Reports of completed research or a major significant phase of research that present the results of NASA programs and include extensive data or theoretical analysis. Includes compilations of significant scientific and technical data and information deemed to be of continuing reference value. NASA counter-part or peer-reviewed formal professional papers, but having less stringent limitations on manuscript length and extent of graphic presentations.

\section{- TECHNICAL MEMORANDUM.}

Scientific and technical findings that are preliminary or of specialized interest, e.g., quick release reports, working papers, and bibliographies that contain minimal annotation. Does not contain extensive analysis.

- CONTRACTOR REPORT. Scientific and technical findings by NASA-sponsored contractors and grantees.
- CONFERENCE PUBLICATIONS. Collected papers from scientific and technical conferences, symposia, seminars, or other meetings sponsored or co-sponsored by NASA.

- SPECIAL PUBLICATION. Scientific, technical, or historical information from NASA programs, projects, and missions, often concerned with subjects having substantial public interest.

- TECHNICAL TRANSLATION. Englishlanguage translations of foreign scientific and technical material pertinent to NASA's mission.

Specialized services that help round out the STI Program Office's diverse offerings include creating custom thesauri, building customized databases, organizing and publishing research results ... even providing videos.

For more information about the NASA STI Program Office, you can:

- Access the NASA STI Program Home Page at http://www.sti.nasa.gov/STIhomepage.html

- Email your question via the Internet to help@sti.nasa.gov

- Fax your question to the NASA Access Help Desk at (301) 621-0134

- Phone the NASA Access Help Desk at (301) 621-0390

- Write to: NASA Access Help Desk NASA Center for AeroSpace Information 800 Elkridge Landing Road Linthicum Heights, MD 21090-2934 
NASA/CR-97-206269

ICASE Report No. 97-69

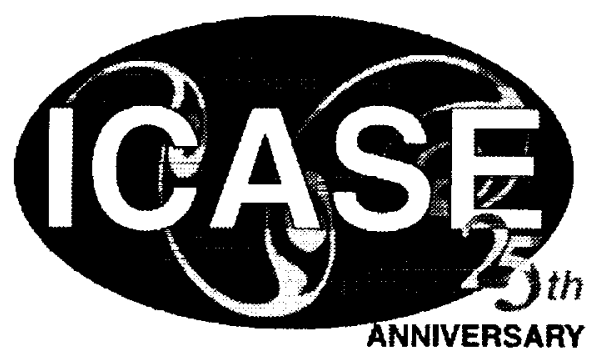

\section{Admitting the Inadmissible: Adjoint Formulation for Incomplete Cost Functionals in Aerodynamic Optimization}

Eyal Arian and Manuel D. Salas

ICASE

Institute for Computer Applications in Science and Engineering NASA Langley Research Center

Hampton, VA

Operated by Universities Space Research Association

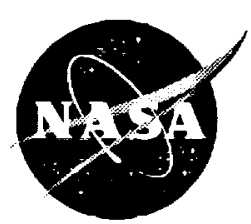

National Aeronautics and

Space Administration

Langley Research Center

Hampton, Virginia 23681-2199 
Available from the following:

NASA Center for AeroSpace Information (CASI)

800 Elkridge Landing Road

Linthicum Heights, MD 21090-2934

(301) 621-0390
National Technical Information Service (NTIS) 5285 Port Royal Road

Springfield, VA 22161-2171

(703) $487-4650$ 


\title{
ADMITTING THE INADMISSIBLE: ADJOINT FORMULATION FOR INCOMPLETE COST FUNCTIONALS IN AERODYNAMIC OPTIMIZATION *
}

\author{
EYAL ARIAN ${ }^{\dagger}$ AND MANUEL D. SALAS ${ }^{\ddagger}$
}

\begin{abstract}
We derive the adjoint equations for problems in aerodynamic optimization which are improperly considered as "inadmissible". For example, a cost functional which depends on the density, rather than on the pressure, is considered "inadmissible" for an optimization problem governed by the Euler equations. We show that for such problems additional terms should be included in the Lagrangian functional when deriving the adjoint equations. These terms are obtained from the restriction of the interior PDE to the control surface. Demonstrations of the explicit derivation of the adjoint equations for "inadmissible" cost functionals are given for the potential, Euler, and Navier-Stokes equations.
\end{abstract}

Key words. adjoint, aerodynamic optimization, Euler, Navier-Stokes, optimal shape.

Subject classification. Applied and Numerical Mathematics

1. Introduction. In recent years there has been a growing interest in solving optimization problems governed by the Euler and the Navier-Stokes (NS) equations (for example [1]-[10]). The new interest in this classical field is due to advances in computer performance and improvements in algorithms for the numerical solution of the flow equations. Among the many optimization methods that are being pursued, the Lagrange multiplier method or adjoint method is particularly attractive, because of its efficiency for problems with many design variables. The adjoint method is based on a variation analysis of the Lagrangian and requires that the variation vanishes at the optimum. This necessary condition yields an optimality system of coupled PDEs consisting of the state equation, the costate or adjoint equation, the optimality condition, and boundary conditions for the state and costate equations.

Recently, Anderson and Venkatakrishnan [8] reported some difficulties in the derivation of boundary conditions for the costate equation for certain cost functionals. The same problem was later reported in [9][10]. In [8], for example, it is concluded that for aerodynamic optimization problems which are governed by the compressible Euler flow equations, only cost functionals that depend solely on the pressure, $F=F(p)$, are admissible; and for viscous flow, using the compressible (NS) equations, only cost functionals which involve the entire stress tensor (e.g., drag) are admissible. As stated in [8], the difficulty with the "inadmissible" cost functionals stems from the need for a suitable balance between the different terms in the variational form of the Lagrangian; for some cost functionals such a balance does not exist and the requirement that the variation of the Lagrangian vanish does not result in a boundary value problem for the adjoint variables. In [9], the authors suggest, for the compressible NS with adiabatic boundary condition on the solid wall, to introduce a contribution into the cost functional which depends on the temperature so that appropriate cancellation in the variation of the Lagrange functional will occur. In [10], the authors concluded that no other choices of cost functionals, other than those suggested in [8], lead to a well-posed problem.

From the theory of functional analysis costate variables exist for all cost functionals [11]. This, however,

*This research was supported by the National Aeronautics and Space Administration under NASA Contract No. NAS197046 while the authors were in residence at the Institute for Computer Applications in Science and Engineering (ICASE), M/S 403, NASA Langley Research Center, Hampton, VA, 23681-0001.

$\dagger$ ICASE, M/S 403, NASA Langley Research Center, Hampton, VA 23681-0001 (email: arian Qicase.edu).

$\ddagger$ ICASE, M/S 403, NASA Langley Research Center, Hampton, VA 23681-0001 (email: salas@icase.edu). 
is not the same as saying that all cost functionals lead to a proper boundary value problem for the costate equation, which is what we mean by an "admissible" cost functional.

In this paper, a general method is presented for formulating proper boundary value problems from cost functionals considered "inadmissible" in the literature. The method avoids redefining, or introducing new terms in, the cost functional. The term "inadmissible" is obviously incorrect. We claim that for so called "inadmissible" cost functionals additional auxiliary boundary equations are needed in the Lagrangian. These relations are obtained from the restriction of the interior PDE and its derivatives (up to the highest order possible) to the boundary. With these additional relations, proper cancellation of terms in the variation of the Lagrange functional can be obtained for any well defined cost functional. However, there is value in distinguishing those cost functionals that lead to a proper boundary value problem with out the need of auxiliary boundary equations from those that do not. To that end, we define complete-cost functionals as those that lead to a well posed boundary value problem of the costate equation with out need for augmenting the Lagrangian functional with auxiliary boundary equations and we define incomplete-cost functionals as those requiring the use of auxiliary boundary equations.

The paper is organized as follows. In $\S 2$ we begin with the potential equation and an admissible cost functional. This example is intended to illustrate the problem that arises later with the cost functional containing $\frac{\partial^{2} \phi}{\partial n^{2}}$ which is also treated in this section. In $\S 3$ the adjoint equations are derived for a cost functional containing the density, $\rho$, for shape optimization problems governed by the compressible Euler equations. In $\S 4$ the adjoint equations are derived for a cost functional containing only the pressure, $p$, for shape optimization problems governed by the compressible NS equations. In $\S 5$ we discuss our findings and make some concluding remarks. Appendix A contains the definition of the Euler Jacobian matrices. Appendix B contains identities of polar coordinates which are used extensively in $\S \S 3-4$. Appendix $\mathrm{C}$ contains a demonstration of the adjoint derivation on a more complex cost functional, than the one presented in $\S 4$, for shape optimization problems governed by the compressible NS equations.

2. The Potential Equation. Let $\Omega$ be a two dimensional domain confined in the area between two circles with radii $R_{1}<R_{2}$. We denote by $\Gamma$ the circle with radius $R_{1}$ and by $\partial \Omega-\Gamma$ the circle with radius $R_{2}$. Let $f^{*}(\xi)$ be a given $L^{2}(\Gamma)$ function defined on the boundary $\Gamma$, and let the function $\alpha(\xi)$, defined on $\Gamma$, be the design variable.

2.1. An "Admissible" Cost Functional. Consider first the derivation of the adjoint equation on a Dirichlet cost functional using the standard procedure. The minimization problem is defined as follows:

$$
\min _{\alpha} F_{1}(\phi)=\int_{\Gamma}\left(\phi-f^{*}\right)^{2} d \sigma
$$

subject to

$$
\begin{array}{cl}
\Delta \phi=0 & \text { in } \Omega \\
\frac{\partial \phi}{\partial n}=\frac{\partial \alpha}{\partial t} & \text { on } \Gamma \\
\phi=\phi_{0} & \text { on } \partial \Omega-\Gamma
\end{array}
$$

where $n$ and $t$, respectively, denote the outer normal and tangential directions on the boundary $\Gamma$. The definition of the state equation (2.2) assumes the following smoothness properties:

$$
\begin{gathered}
\alpha \in H^{1}(\Gamma) \\
\phi \in H^{\frac{3}{2}}(\Omega),
\end{gathered}
$$


where $H^{k}$ denotes the Sobolev space of order $k$ (see [12]). The necessary conditions for a minimum are derived with the adjoint method. We introduce the Lagrange multipliers $\lambda$ defined on the domain $\Omega, \zeta$ defined on part of the boundary $\Gamma$, and $\vartheta$ defined on the rest of the boundary $\partial \Omega-\Gamma$. In terms of the Lagrange multipliers, a Lagrangian is defined by:

$$
\mathcal{L}(\phi, \alpha, \lambda, \zeta, \vartheta)=\int_{\Gamma}\left(\phi-f^{*}\right)^{2} d \sigma-\int_{\Omega}(\lambda \Delta \phi) d \Omega+\int_{\Gamma} \zeta\left(\frac{\partial \phi}{\partial n}-\frac{\partial \alpha}{\partial t}\right) d \sigma+\int_{\partial \Omega-\Gamma} \vartheta\left(\phi_{0}-\phi\right) d \sigma
$$

The variation of the Lagrangian is given by:

$$
\begin{aligned}
& (2.5 \mathrm{a}) \tilde{\mathcal{L}}=\int_{\Gamma} 2 \tilde{\phi}\left(\phi-f^{*}\right) d \sigma-\int_{\Omega} \tilde{\phi} \Delta \lambda d \Omega+\int_{\partial \Omega}\left(\tilde{\phi} \frac{\partial \lambda}{\partial n}-\lambda \frac{\partial \tilde{\phi}}{\partial n}\right) d \sigma+\int_{\Gamma} \zeta\left(\frac{\partial \tilde{\phi}}{\partial n}-\frac{\partial \tilde{\alpha}}{\partial t}\right) d \sigma+\int_{\partial \Omega-\Gamma} \vartheta \tilde{\phi} d \sigma \\
& (2.5 \mathrm{~b})=-\int_{\Omega} \tilde{\phi} \Delta \lambda d \Omega+\int_{\Gamma} \tilde{\phi}\left(\frac{\partial \lambda}{\partial n}+2\left(\phi-f^{*}\right)\right) d \sigma+\int_{\Gamma} \frac{\partial \tilde{\phi}}{\partial n}(\zeta-\lambda) d \sigma+\int_{\partial \Omega-\Gamma} \frac{\partial \tilde{\phi}}{\partial n} \lambda d \sigma+\int_{\Gamma} \tilde{\alpha} \frac{\partial \zeta}{\partial t} d \sigma .
\end{aligned}
$$

The term containing $\tilde{\phi}$ on $\partial \Omega-\Gamma$ was omitted since $\phi$ is fixed on that part of the boundary (see Eq.(2.2)) and therefore its variation vanishes.

The necessary conditions for a minimum are obtained by requiring that the integrands in $(2.5 \mathrm{~b})$ vanish. Matching the terms that multiply $\tilde{\phi}$, and those that multiply $\frac{\partial \tilde{\phi}}{\partial n}$, results in the following equations:

$$
\begin{array}{cc}
\tilde{\phi}(\Omega): & \Delta \lambda=0 \text { in } \Omega \\
\tilde{\phi}(\Gamma): & \frac{\partial \lambda}{\partial n}=-2\left(\phi-f^{*}\right) \text { on } \Gamma \\
\frac{\partial \tilde{\phi}}{\partial n}(\Gamma): & \zeta=\lambda \text { on } \Gamma \\
\frac{\partial \tilde{\phi}}{\partial n}(\partial \Omega-\Gamma): & \lambda=0 \text { on } \partial \Omega-\Gamma \\
\tilde{\alpha}(\Gamma): & \frac{\partial \zeta}{\partial t}=0 \text { on } \Gamma .
\end{array}
$$

Therefore, the costate (adjoint) boundary value problem is defined by

$$
\begin{gathered}
\Delta \lambda=0 \quad \text { in } \Omega \\
\frac{\partial \lambda}{\partial n}=-2\left(\phi-f^{*}\right) \quad \text { on } \Gamma \\
\lambda=0 \quad \text { on } \partial \Omega-\Gamma,
\end{gathered}
$$

with the Fréchet derivative of the cost functional with respect to $\alpha$ given by

$$
\frac{d F_{1}}{d \alpha}=\frac{\partial \lambda}{\partial t} \text { on } \Gamma
$$

2.2. An "Inadmissible" Cost Functional. Suppose that we want to minimize a cost functional that depends on the second normal derivative on the boundary. Then the minimization problem is defined as follows:

$$
\min _{\alpha} F_{2}(\phi)=\int_{\Gamma}\left(\frac{\partial^{2} \phi}{\partial n^{2}}-f^{*}\right)^{2} d \sigma
$$

subject to Eq.(2.2). However, the definition of the cost functional (2.8) assumes that the second normal derivative of $\phi$ on the boundary exists and is in $L^{2}(\Gamma)$. This is not consistent with the smoothness requirement of $\phi,(2.3)$, unless we additionally assume that $\alpha$ is smoother than required by the state PDE, i.e.,

$$
\begin{gathered}
\alpha \in H^{2}(\Gamma) \\
\phi \in H^{\frac{5}{2}}(\Omega) .
\end{gathered}
$$


If we derive the necessary conditions for a minimum as we did for the Dirichlet cost functional (2.1), then the variation of the Lagrangian is given by:

$$
\tilde{\mathcal{L}}=\int_{\Gamma} 2 \frac{\partial^{2} \tilde{\phi}}{\partial n^{2}}\left(\frac{\partial^{2} \phi}{\partial n^{2}}-f^{*}\right) d \sigma-\int_{\Omega}(\tilde{\phi} \Delta \lambda) d \Omega+\int_{\partial \Omega}\left(\tilde{\phi} \frac{\partial \lambda}{\partial n}-\lambda \frac{\partial \tilde{\phi}}{\partial n}\right) d \sigma+\int_{\Gamma} \zeta\left(\frac{\partial \tilde{\phi}}{\partial n}-\frac{\partial \tilde{\alpha}}{\partial t}\right) d \sigma
$$

(As previously discussed, the term that contains $\tilde{\phi}$ on the "far-field", $\partial \Omega-\Gamma$, was omitted.)

In the previous example, we saw how the first and third terms in the variation of the Lagrangian (2.5a) combined to give a boundary condition for $\frac{\partial \lambda}{\partial n}$ along $\Gamma$. In this example, the first term in (2.10), the term with $\frac{\partial^{2} \bar{\phi}}{\partial n^{2}}$, can not be combined with any of the other terms on the boundary $\Gamma$ and, hence, we can not obtain a boundary condition on $\Gamma$ for the costate equation. A cost functional exhibiting this behavior was termed "inadmissible" in the literature [8]. However, since the state equation (2.2) is linear, $\phi$ depends on $\alpha$ linearly, the cost functional $F_{2}$ is a quadratic in $\alpha$. A quadratic cost functional has a unique minimizer, thus the cost functional $F_{2}$ is in-fact admissible.

In the next subsection, we show how to overcome this problem using an auxiliary boundary equation.

2.3. Auxiliary Boundary Equation (ABE). For convenience, let us introduce polar coordinates $(r, \theta)$. In polar coordinates, the restriction of the interior PDE (2.2) to the boundary $\Gamma$ results in the following ABE (assuming the consistency requirements of (2.9))

$$
\frac{1}{R_{1}} \frac{\partial \phi}{\partial r}+\frac{\partial^{2} \phi}{\partial r^{2}}+\frac{1}{R_{1}^{2}} \frac{\partial^{2} \phi}{\partial \theta^{2}}=0 \text { on } \Gamma \text {. }
$$

Here we assume that $\hat{r}=-\hat{n}$ is perpendicular to the boundary, pointing into the domain, and $R_{1}$ is the radius of curvature. By Eq. $(2.11)$ we get $\left(d t=R_{1} d \theta\right)$

$$
\frac{\partial^{2} \bar{\phi}}{\partial r^{2}}=\left(-\frac{1}{R_{1}} \frac{\partial \tilde{\phi}}{\partial r}-\frac{\partial^{2} \tilde{\phi}}{\partial t^{2}}\right) \text { on } \Gamma \text {, }
$$

which can be used to replace $\frac{\partial^{2} \bar{\phi}}{\partial n^{2}}$ in the variation of the Lagrangian. Integration by parts, along the boundary, of the term containing tangential derivatives results in a variational form of the Lagrangian which contains only the naturally occurring boundary terms $\left(\tilde{\phi}\right.$ and $\left.\frac{\partial \tilde{\phi}}{\partial r}\right)$.

Another way to use Eq.(2.11), which leads to the same result, is by adding it to the Lagrangian with a new Lagrange multiplier $\eta$. Then $\tilde{\mathcal{L}}$ is augmented with the term $\int_{\Gamma} \eta\left(-\frac{1}{R_{1}} \frac{\partial \bar{\phi}}{\partial r}-\frac{\partial^{2} \tilde{\phi}}{\partial r^{2}}-\frac{\partial^{2} \tilde{\phi}}{\partial t^{2}}\right) d \sigma$ which results in the following adjoint equations on the boundary:

$$
\begin{array}{cc}
\frac{\partial^{2} \bar{\phi}}{\partial r^{2}}(\Gamma): & -\eta+2\left(\frac{\partial^{2} \phi}{\partial r^{2}}-f^{*}\right)=0 \\
\tilde{\phi}(\Gamma): & -\frac{\partial^{2} \eta}{\partial t^{2}}-\frac{\partial \lambda}{\partial r}=0 \\
-\frac{\partial \tilde{\phi}}{\partial r}(\Gamma): & \frac{1}{R_{1}} \eta-\lambda+\zeta=0 .
\end{array}
$$

Therefore, the adjoint equations can be written in the following "strong" form:

$$
\begin{gathered}
\Delta \lambda=0 \text { in } \Omega \\
-\frac{\partial \lambda}{\partial r}=2\left(\frac{\partial^{4} \phi}{\partial r^{2} \partial t^{2}}-\frac{\partial^{2} f^{*}}{\partial t^{2}}\right) \quad \text { on } \Gamma \\
\lambda=0 \text { on } \partial \Omega-\Gamma .
\end{gathered}
$$

The Fréchet derivative of the cost functional $F_{2}$ with respect to $\alpha$ is then given by

$$
\frac{d F_{2}}{d \alpha}=\frac{\partial \zeta}{\partial t}=\frac{\partial \lambda}{\partial t}-\frac{2}{R_{1}}\left(\frac{\partial^{3} \phi}{\partial r^{2} \partial t}-\frac{\partial f^{*}}{\partial t}\right) \quad \text { on } \Gamma .
$$


In the same manner other cost functionals can be treated by taking (if necessary and consistent with the smoothness assumption of the cost functional definition) higher derivatives of, $\Delta \phi=0$, restricting the resulting equations to the boundary and adding them to the Lagrangian with additional Lagrange multipliers.

We add a remark on the required smoothness of the Lagrange multipliers. If we insist in solving the adjoint equation in its "strong" form (2.14), then the smoothness requirement of the state solution should be even stronger than (2.9) (also $f^{*}(s)$ should be smoother than $L^{2}(\Gamma)$ ). This requirement can be relaxed if the adjoint equations are solved in a weak formulation, for example, by finite elements [11]. For future reference we give here the following definition.

DEFINITION 2.1. We define a complete-cost functional as one that leads to a boundary value problem of the costate equation with out the need for augmenting the Lagrangian functional with auxiliary boundary equations. Otherwise, the cost functional will be termed as incomplete.

In the next sections we treat similarly higher level models of fluid dynamics PDEs. In these cases, the functional analysis of the smoothness properties of the state equations are not as obvious as in the above example.

3. The Euler Equations. For simplicity the derivation is done in two dimensions. Let $\vec{U}$ denote the vector of state variables:

$$
\vec{U}=(\rho, \rho u, \rho v, \rho E)^{T}
$$

where we use the following notation: $\rho$ denotes the density, $\vec{u}=(u, v)$ denotes the velocity vector, and $E$ denotes the total specific energy.

The Euler equations are given by (conservative form)

$$
\begin{gathered}
\operatorname{div}(\rho \vec{u})=0 \\
\operatorname{div}(\rho \vec{u} \otimes \vec{u}+p I)=0 \\
\operatorname{div}(\rho \vec{u} H)=0
\end{gathered}
$$

where $\vec{u} \cdot \vec{n}=0$ on the solid wall $\Gamma$, and with additional appropriate boundary conditions on the far-field. (The above system, in the interior, can be written in an equivalent form in terms of the Jacobian matrices, $\nabla(\vec{A} \vec{U})=0$, where $\vec{A}=(A, B)$ denote the Jacobian matrices given in appendix A.)

The following are state relations for the pressure, $p$, and the total enthalpy, $H$ :

$$
\begin{gathered}
p=(\gamma-1) \rho E-\frac{\gamma-1}{2} \rho|\vec{u}|^{2} \\
H=\frac{\gamma}{\gamma-1} \frac{p}{\rho}+\frac{|\vec{u}|^{2}}{2} .
\end{gathered}
$$

3.1. Natural Boundary Terms. Integrating by parts the Euler equations results in a term containing the pressure on the boundary:

$$
\int_{\Omega} \nabla(\vec{A} \vec{U}) d \Omega=\int_{\partial \Omega} p\left(0, n_{1}, n_{2}, 0\right)^{T} d \sigma
$$

(in polar coordinates $n_{1}=-1$ and $n_{2}=0$ ), therefore any minimization problem that contains terms other than the pressure will result in non-canceling terms in the variational Lagrangian.

3.2. Example of an Incomplete Cost Functional. The following cost functional is incomplete (the design variable is the shape of the solid wall $\Gamma$ ):

$$
F(\rho)=\int_{\Gamma}\left(\rho-\rho^{*}\right)^{2} d \sigma
$$


The Lagrangian is given by

$$
\mathcal{L}=\int_{\Gamma}\left(\rho-\rho^{*}\right)^{2} d \sigma+\int_{\Omega} \vec{\Lambda}^{T} \nabla(\vec{A} \vec{U}) d \Omega+\int_{\Gamma} \zeta(\vec{u} \cdot \vec{n}) d \sigma+\text { far-field terms }
$$

where $\vec{\Lambda}$ is the vector of costate variables,

$$
\vec{\Lambda}=\left(\lambda_{1}, \lambda_{2}, \lambda_{3}, \lambda_{4}\right)^{T}
$$

and we denote by $\vec{\lambda}$ the adjoint "velocity" vector, i.e.,

$$
\vec{\lambda}=\left(\lambda_{2}, \lambda_{3}\right) \text {. }
$$

The variation of the Lagrangian is given by

$$
\tilde{\mathcal{L}}=\int_{\Gamma} 2 \tilde{\rho}\left(\rho-\rho^{*}\right) d \sigma+\int_{\Omega} \tilde{\vec{U}}\left(A^{T} \vec{\Lambda}_{x}+B^{T} \vec{\Lambda}_{y}\right) d \Omega+\int_{\Gamma} \tilde{p} \lambda_{2} d \sigma+\int_{\Gamma} \tilde{\alpha} g(\vec{U}, \vec{\Lambda}) d \sigma+\text { far-field terms, }
$$

which results in non-cancellation of terms in $\tilde{\mathcal{L}}$, since the cost functional is not given in terms of the natural boundary term $p^{1}$.

However, in general we can write

$$
\rho=\rho(p, s)
$$

where $s$ is the entropy and, in particular, on the surface $\Gamma$ we can write

$$
\rho=\rho(p)
$$

in the absence of shock waves ${ }^{2}$, since the entropy is constant along the streamline wetting the surface. Hence, we know that we can overcome this problem. In the next section we derive the adjoint equations, for the incomplete cost functional (3.5), with a general procedure, along the lines of $\S 2.3$.

3.3. Auxiliary Boundary Equations. As in the potential problem we derive auxiliary boundary equations (ABEs) by restricting the interior PDEs to the boundary. For simplicity, we examine the resulting equation locally around a point on the boundary and use polar coordinates. Fig.(1) depicts the local coordinate system. Throughout the paper we use the unit tangential vector $\vec{t}$ on the boundary instead of $\vec{\theta}$. Note that on the boundary, $\Gamma$,

$$
\frac{\partial}{\partial t}=\frac{1}{R} \frac{\partial}{\partial \theta}
$$

Also, in polar coordinates the components of the velocity vector $\vec{u}$ will be denoted by $\vec{u}=\left(u_{r}, u_{t}\right)$ where $u_{r}=\vec{u} \cdot \vec{r}$ and $u_{t}=\vec{u} \cdot \vec{\theta}$; similarly, the components of the adjoint "velocity" vector $\vec{\lambda}$ will be denoted by $\vec{\lambda}=\left(\lambda_{r}, \lambda_{t}\right)$ where $\lambda_{r}=\vec{\lambda} \cdot \vec{r}$ and $\lambda_{t}=\vec{\lambda} \cdot \vec{\theta}$.

3.3.1. The Continuity Equation. In polar coordinates, on the boundary, the continuity equation is given by $(\nabla(\rho \vec{u})=0)$

$$
\frac{\partial}{\partial r}\left(\rho u_{r}\right)+\frac{\partial}{\partial t}\left(\rho u_{t}\right)=0 .
$$

Also higher order derivatives of the continuity equation can be taken and restricted to the boundary and are considered auxiliary boundary equations, as long as the solution in the interior is smooth enough so that these derivatives exist.

\footnotetext{
${ }^{1}$ In fact, for shape optimization problems, as we discuss here, the variation of the Lagrangian includes more terms on the boundary $\Gamma$ that depend on $\overline{\vec{u}} \cdot \vec{n}$. However, these terms contribute only to the gradient term $\int_{\Gamma} \bar{\alpha} g(\vec{U}, \vec{\Lambda}) d \sigma$ and therefore do not play a role in the derivation of the adjoint boundary value problem. For simplicity we do not discuss these terms in this paper.

${ }^{2}$ In the presence of shocks, it is still valid to write $\rho=\rho(p)$ in a piecewise sense between shocks using the Rankine-Hugoniot conditions to connect the piecewise regions along the streamline wetting the surface.
} 


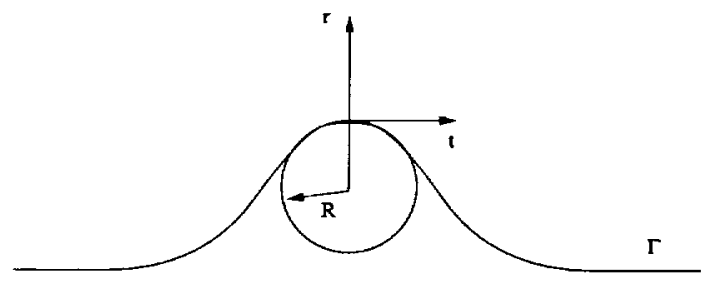

FIG. 1. The local coordinate system around a point on the boundary.

3.3.2. The Momentum Equations. The momentum equations, $\nabla \cdot(\rho \vec{u} \otimes \vec{u}+p I)=0$, in polar coordinates are given by (see Eq.(B.4))

$$
\begin{gathered}
\frac{\partial}{\partial r}\left(\rho u_{r}^{2}\right)+\frac{\rho u_{r}^{2}}{r}+\frac{1}{r} \frac{\partial}{\partial \theta}\left(\rho u_{r} u_{t}\right)-\frac{\rho u_{t}^{2}}{r}+\frac{\partial p}{\partial r}=0 \\
\frac{\partial}{\partial r}\left(\rho u_{r} u_{t}\right)+2 \frac{e u_{r} u_{t}}{r}+\frac{1}{r} \frac{\partial}{\partial \theta}\left(\rho u_{t}^{2}\right)+\frac{\partial p}{\partial t}=0 .
\end{gathered}
$$

Using the solid wall boundary condition and the relation (3.13), the restriction of the above to the boundary results in

$$
\begin{gathered}
-\frac{\rho u_{t}^{2}}{R}+\frac{\partial p}{\partial r}=0 \\
\rho u_{t} \frac{\partial u_{t}}{\partial t}+\frac{\partial p}{\partial t}=0,
\end{gathered}
$$

where $R$ denotes the local radius of curvature (see Fig. (1)).

Higher order equations can be derived by taking derivatives of equations (3.14) and restricting them to the boundary.

3.3.3. The Energy Equation. The energy equation in polar coordinates is given by $(\nabla \cdot(\rho \vec{u} H)=0)$

$$
\frac{1}{r} \frac{\partial}{\partial \theta}\left(\rho u_{t} H\right)+\frac{1}{r} \frac{\partial}{\partial r}\left(\rho r u_{r} H\right)=0 .
$$

Using the solid wall boundary condition and the relation (3.13), the restriction of the above to the boundary results in

$$
\frac{\partial H}{\partial t}=0 \quad \text { or } \quad H=\text { const on } \Gamma .
$$

3.3.4. The Derivation of the Adjoint Equations. The definition of the cost functional (3.5) contains an implicit assumption that the restriction of the density state variable to the solid wall is continuous (and also that $\rho(\Gamma) \in L^{2}(\Gamma)$ ). However, in general the density is not continuous in the direction perpendicular to streamlines while the pressure, $p$, and the normal velocity, $u_{r}$, are always continuous in that direction [13]. The new assumption on the smoothness of the density is introduced into the Lagrangian by adding the ABEs:

$$
\mathcal{L}_{A B E}=\int_{\Gamma}\left[\eta_{1}\left(\frac{\partial}{\partial n}(\rho \vec{u} \cdot \vec{n})+\frac{\partial}{\partial t}(\rho \vec{u} \cdot \vec{t})\right)+\eta_{2}\left(-\frac{\rho u_{2}^{2}}{r}+\frac{\partial p}{\partial r}\right)+\eta_{3}\left(\rho u_{t} \frac{\partial u_{t}}{\partial t}+\frac{\partial p}{\partial t}\right)+\eta_{4} H\right] d \sigma
$$

where $\left(\eta_{1}, \cdots, \eta_{4}\right)$ are additional Lagrange multipliers. For the cost functional (3.5), the restriction of the continuity and first momentum equations are not required and therefore we choose $\eta_{1}=\eta_{2}=0$. The variation of the enlarged Lagrangian, $\mathcal{L}+\mathcal{L}_{A B E}$, yields three adjoint equations on the boundary for $\vec{\lambda}, \eta_{3}$ and $\eta_{4}$ :

$$
\begin{array}{cc}
\tilde{\rho}(\Gamma): & \eta_{3} u_{t} \frac{\partial u_{t}}{\partial t}-\eta_{4} \frac{\gamma}{\gamma-1} \frac{p}{\rho^{2}}+2\left(\rho-\rho^{*}\right)=0 \\
\tilde{u}_{t}(\Gamma): & \eta_{3} \rho \frac{\partial u_{t}}{\partial t}-\frac{\partial}{\partial t}\left(\rho u_{t} \eta_{3}\right)+\eta_{4} u_{t}=0 \\
\tilde{p}(\Gamma): & -\frac{\partial \eta_{3}}{\partial t}+\frac{\gamma}{\gamma-1} \eta_{4} \frac{1}{\rho}+\lambda_{r}=0
\end{array}
$$


where we have used the relation ${ }^{3}$

$$
\tilde{H}=\frac{\gamma}{\gamma-1}\left(\frac{\tilde{p}}{\rho}-\frac{p}{\rho^{2}} \tilde{\rho}\right)+u_{t} \tilde{u}_{t}=0 .
$$

The above system can be solved by solving the first two PDEs in (3.19) for $\eta_{3}$ and $\eta_{4}$ and substituting the result in the third equation which is the transpiration boundary condition for $\vec{\lambda}$.

4. The Navier-Stokes Equations. The compressible NS equations are given by

$$
\begin{gathered}
\operatorname{div}(\rho \vec{u})=0 \\
\operatorname{div}(\rho \vec{u} \otimes \vec{u})=\operatorname{div}(\sigma) \\
\operatorname{div}(e \vec{u})+\operatorname{div}(\vec{q})=\operatorname{div}(\sigma \vec{u})
\end{gathered}
$$

where the stress tensor, $\sigma$, is given by

$$
\sigma=-p I+\mu_{2} \operatorname{div}(\vec{u}) I+\mu d e f(\vec{u}),
$$

and $I$ denotes the unit tensor, def $=\operatorname{grad}+\operatorname{grad}^{T}$, and $\mu$ and $\mu_{2}$ are the first and second viscosities, respectively $\left(\mu_{2}+\frac{2}{3} \mu=0\right)$. The vector $\vec{q}$ denotes the heat conduction vector, $\vec{q}=-\operatorname{kgrad}(T)$, where $T$ denotes the temperature, and $k$ denotes the coefficient of conductivity and will be set equal to a constant. The total energy satisfies $e=\rho \frac{|\vec{u}|^{2}}{2}+\rho \frac{T}{\gamma(\gamma-1)}$.

The solid wall boundary conditions are given by

$$
\begin{gathered}
\vec{u}=0 \\
a T+b \frac{\partial T}{\partial n}=c
\end{gathered}
$$

where $a, b$ and $c$ are parameters (in this paper we set $a=c=0$ and $b=1$, resulting in the adiabatic wall boundary condition).

4.1. Natural Boundary Terms. For simplicity we will denote the system of NS equations by $\nabla \cdot \vec{F}=\mathbf{0}$. where $\vec{F}$ consists of the flux vectors. Integration by parts of the compressible NS equations results in the following boundary terms:

$$
\int_{\Omega}(\nabla \cdot \vec{F}) d \Omega=\int_{\partial \Omega}\left(0,(\sigma \vec{n})_{1},(\sigma \vec{n})_{2}, 0\right)^{T} d \sigma
$$

Therefore, the natural boundary terms for the compressible NS equations are the total fluid force components, $(\sigma \vec{n})_{j}$. (In other words, the only complete cost functionals are those which measure lift or drag.)

4.2. Example of an Incomplete Cost Functional. Let us take, for example, the following cost functional which is incomplete (here, as in the previous section, the design variable is the shape $\Gamma$ ) since its variation is not given in terms of the force components in (4.3):

$$
F(p)=\int_{\Gamma}\left(p-p^{*}\right)^{2} d \sigma
$$

The cost functional (4.4) was treated previously in [14] by neglecting a term in the Lagrangian and in [8] by modifying the cost functional. A demonstration of the adjoint derivation on a more complex cost functional is given in appendix $\mathrm{C}$.

\footnotetext{
${ }^{3}$ we assume that the term defined on the endpoints of $\Gamma$ which results from the integration by parts, $\int_{\Gamma} \eta_{3} \frac{\partial p}{\partial t} d \sigma$, is equal to zero, i.e., $\eta_{3} p\left(\partial \Gamma^{+}\right)-\eta_{3} p\left(\partial \Gamma^{-}\right)=0$.
} 
The Lagrangian is given by

$$
\mathcal{L}=\int_{\Gamma}\left(p-p^{*}\right)^{2} d \sigma+\int_{\Omega} \vec{\Lambda}^{T} \cdot(\nabla \cdot \vec{F}) d \Omega+\int_{\Gamma} \zeta \frac{\partial T}{\partial n} d \sigma+\int_{\Gamma} \vec{\vartheta} \cdot \vec{u} d \sigma+\text { far-field terms }
$$

The variation of the Lagrangian is given by

$$
\tilde{\mathcal{L}}=\text { interior terms }+\int_{\Gamma} 2 \tilde{p}\left(p-p^{*}\right) d \sigma+
$$

$$
\int_{\partial \Omega}\left[-\tilde{T} k \frac{\partial \lambda_{4}}{\partial r}+\frac{\partial \tilde{T}}{\partial r} k \lambda_{4}+\frac{\partial \tilde{u}_{r}}{\partial r} \frac{4}{3} \mu \lambda_{r}+\frac{\partial \tilde{u}_{t}}{\partial r} \mu \lambda_{t}+\tilde{p} \lambda_{r}\right] d \sigma+\int_{\Gamma} \tilde{\alpha} g(\vec{U}, \vec{\Lambda}) d \sigma .
$$

The term $\frac{\partial \tilde{T}}{\partial r}$ does not contribute to the adjoint equations (assuming adiabatic boundary conditions) and therefore will be omitted (by the same reasoning the term containing the variation of $\vec{u}$ on $\Gamma$ and the variation of the far-field terms vanish). Also note that the variation in $T$ can be transformed to variations in $p$ and $\rho$ using the equation of state, $T=\frac{p}{(\gamma-1) \rho}$. The cost functional (4.4) is considered incomplete since the term $\frac{\partial \tilde{u}_{r}}{\partial r}$ determines the adjoint boundary condition $\lambda_{r}=0$. As a result we do not obtain a boundary value problem representation of the adjoint equations.

\subsection{Auxiliary Boundary Equations.}

4.3.1. The Continuity Equation. Since the boundary condition on $\Gamma$ implies that $\frac{\partial}{\partial t}\left(\rho u_{t}\right)=0$, we get from the continuity equation that (see Eq.(3.13))

$$
\frac{\partial u_{r}}{\partial r}=0
$$

4.3.2. The Momentum Equations. Let us write the momentum equations in the form

$$
\operatorname{div}(\rho \vec{u} \otimes \vec{u})=\operatorname{div}(\sigma)=\operatorname{div}(-p I)+\operatorname{div}(\tau)
$$

The term $\operatorname{div}(\rho \vec{u} \otimes \vec{u}+p I)$ is given in polar coordinates in Eq.(3.14). Taking the limit to the boundary we get that

$$
\lim _{\vec{x} \rightarrow \Gamma} \operatorname{div}(\rho \vec{u} \otimes \vec{u})=0 .
$$

The term $\tau$ in polar coordinates is given by

$$
\tau=\mu_{2} \operatorname{div}(\vec{u}) I+\mu \operatorname{def}(\vec{u})=\left(\begin{array}{cc}
\mu_{2} \operatorname{div}(\vec{u})+2 \mu \frac{\partial u_{r}}{\partial r} & \mu\left(\frac{\partial u_{r}}{\partial t}-\frac{u_{t}}{r}+\frac{\partial u_{t}}{\partial r}\right) \\
\mu\left(\frac{\partial u_{r}}{\partial t}-\frac{u_{t}}{r}+\frac{\partial u_{t}}{\partial r}\right) & \mu_{2} \operatorname{div}(\vec{u})+2 \mu\left(\frac{\partial u_{t}}{\partial t}+\frac{u_{r}}{r}\right)
\end{array}\right) .
$$

Evaluating $\operatorname{div}(\tau)$ on the boundary and using the identities

$$
\begin{gathered}
\lim _{\vec{x} \rightarrow \Gamma} \operatorname{div}(\vec{u})=0 \\
\lim _{\vec{x} \rightarrow \Gamma} \frac{\partial}{\partial r} \operatorname{div}(\vec{u})=-\frac{1}{\rho} \frac{\partial \rho}{\partial t} \frac{\partial u_{t}}{\partial r}
\end{gathered}
$$

results in the following auxiliary momentum boundary equations (see Eq.(B.4))

$$
\begin{gathered}
\frac{\partial p}{\partial r}+\mu_{2} \frac{1}{\rho} \frac{\partial \rho}{\partial t} \frac{\partial u_{t}}{\partial r}-2 \mu \frac{\partial^{2} u_{r}}{\partial r^{2}}-\mu \frac{\partial^{2} u_{t}}{\partial r \partial t}=0 \\
\frac{\partial p}{\partial t}-\mu \frac{\partial^{2} u_{t}}{\partial r^{2}}-\frac{1}{R} \mu \frac{\partial u_{t}}{\partial r}=0 .
\end{gathered}
$$


4.3.3. The Energy Equation. The energy equation is given by

$$
\operatorname{div}(\boldsymbol{e} \vec{u})+\operatorname{div}(\vec{q})=\operatorname{div}(\sigma \vec{u}) .
$$

Using the relation

$$
e \vec{u}=\left(\rho \frac{|\vec{u}|^{2}}{2}+\frac{\rho}{\gamma(\gamma-1)} T\right) \vec{u}
$$

and taking its divergence in polar coordinates we get

$$
\lim _{\vec{x} \rightarrow \Gamma} \operatorname{div}(e \vec{u})=0 .
$$

The term $\operatorname{div}(\vec{q})=-k \Delta T$ satisfies (assuming an adiabatic boundary condition)

$$
\lim _{\vec{x} \rightarrow \Gamma} \operatorname{div}(\vec{q})=-k\left(\frac{\partial^{2} T}{\partial r^{2}}+\frac{\partial^{2} T}{\partial t^{2}}\right)
$$

The term $\sigma \vec{u}$ and its tangential derivatives on the boundary are zero, therefore only its radial derivative is considered:

$$
\varliminf_{\vec{x} \rightarrow \Gamma} \operatorname{div}(\sigma \vec{u})=\mu\left(\frac{\partial u_{t}}{\partial r}\right)^{2} .
$$

Eqs.(4.11)-(4.13) imply the following auxiliary energy boundary equation

$$
-k\left(\frac{\partial^{2} T}{\partial r^{2}}+\frac{\partial^{2} T}{\partial t^{2}}\right)-\mu\left(\frac{\partial u_{t}}{\partial r}\right)^{2}=0
$$

4.3.4. The Derivation of the Adjoint Equations. As in the Euler case, we add the ABEs to the Lagrangian:

$$
\begin{gathered}
\mathcal{L}_{A B E}=\int_{\Gamma}\left[\eta_{1}\left(\frac{\partial u_{r}}{\partial r}\right)+\eta_{2}\left(\frac{\partial p}{\partial r}+\mu_{2} \frac{1}{\rho} \frac{\partial \rho}{\partial t} \frac{\partial u_{t}}{\partial r}-2 \mu \frac{\partial^{2} u_{r}}{\partial r^{2}}-\mu \frac{\partial^{2} u_{t}}{\partial r \partial t}\right)\right. \\
\left.+\eta_{3}\left(\frac{\partial p}{\partial t}-\mu \frac{\partial^{2} u_{t}}{\partial r^{2}}-\frac{1}{R} \mu \frac{\partial u_{t}}{\partial r}\right)+\eta_{4}\left(-k\left(\frac{\partial^{2} T}{\partial r^{2}}+\frac{\partial^{2} T}{\partial t^{2}}\right)-\mu\left(\frac{\partial u_{t}}{\partial r}\right)^{2}\right)\right] d \sigma
\end{gathered}
$$

However, for the cost functional (4.4) we need only the first term in Eq.(4.15) and therefore we choose $\eta_{2}=\eta_{3}=\eta_{4}=0$ :

$$
\tilde{\mathcal{L}}+\tilde{\mathcal{L}}_{A B E}=\text { interior terms }+\int_{\Gamma} 2 \tilde{p}\left(p-p^{*}\right) d \sigma+
$$

$$
\int_{\partial \Omega}\left[-\tilde{T} k \frac{\partial \lambda_{4}}{\partial r}+\frac{\partial \tilde{u}_{r}}{\partial r}\left(\eta_{1}+\frac{4}{3} \mu \lambda_{r}\right)+\frac{\partial \tilde{u}_{t}}{\partial r} \mu \lambda_{t}+\tilde{p} \lambda_{r}\right] d \sigma+\int_{\Gamma} \tilde{\alpha} g_{1}\left(\vec{U}, \vec{\Lambda}, \eta_{1}\right) d \sigma
$$

The variation of the enlarged Lagrangian, $\mathcal{L}+\mathcal{L}_{A B E}$, yields the following equations on the boundary (using $\left.\tilde{T}=\frac{1}{\gamma-1}\left(-\frac{1}{\rho^{2}} \tilde{\rho}+\frac{1}{\rho} \tilde{p}\right)\right)$

$$
\begin{array}{cc}
\frac{\partial \tilde{u}_{r}}{\partial r}(\Gamma): & \eta_{1}+\frac{4}{3} \mu \lambda_{r}=0 \\
\tilde{p}(\Gamma): & 2\left(p-p^{*}\right)+\lambda_{r}-\frac{k}{\gamma-1} \frac{1}{\rho} \frac{\partial \lambda_{4}}{\partial r}=0 \\
\frac{\partial \tilde{u}_{t}}{\partial r}(\Gamma): & \mu \lambda_{t}=0 \\
\tilde{\rho}(\Gamma): & \frac{k}{\gamma-1} \frac{1}{\rho^{2}} \frac{\partial \lambda_{4}}{\partial r}=0 .
\end{array}
$$

The resulting adjoint equations on the solid wall are given by (See Eq.(4.2))

$$
\begin{gathered}
\lambda_{T}=-2\left(p-p^{*}\right) \\
\lambda_{t}=0 \\
\frac{d \lambda_{4}}{d n}=0 .
\end{gathered}
$$


Note that the first equation in (4.17) determines the auxiliary Lagrange multiplier $\eta_{1}$ :

$$
\eta_{1}=-\frac{4}{3} \mu \lambda_{r}=\frac{8}{3} \mu\left(p-p^{*}\right)
$$

That Lagrange multiplier is affecting the gradient, $g_{1}=g_{1}\left(\vec{U}, \vec{\Lambda}, \eta_{1}\right)$, since the variation of the continuity auxiliary boundary equation (4.7) with respect to a change in the shape, $\Gamma$, results in variational boundary terms that multiply only the term $\tilde{\alpha}$.

5. Discussion and Concluding Remarks. We present a method for the derivation of the costate equations for problems in which the cost functional does not lead to a proper boundary value problem for the costate equation, when derived in the standard way. We define such cost functionals as incomplete; it is required to "complete" the Lagrangian with auxiliary boundary equations in these cases in order to derive a boundary value problem of the costate equation. We demonstrate the method on three problems involving the incomplete cost functionals using the potential, the compressible Euler, and the compressible NS equations. Our aim is to give the costate equations a representation of a boundary value problem and not to treat rigorously the issue of existence of solutions to the resulting system of costate equations. We note that for all cost functionals it is possible to derive the adjoint equations in the discrete level in the standard manner; the problem of incompleteness exists only in the PDE level. The relation between the costate equations that we derive and the discretely derived costate equations in the limit of mesh-size going to zero is beyond the scope of this paper.

Acknowledgments. The authors thank Dr. Bambang I. Soemarwoto, of the Institute of Technology Bandung in Indonesia, for fruitful discussions on the derivation of the adjoint equations for the Navier-Stokes equations.

\section{REFERENCES}

[1] B. Mantel, J. Periaux, B. Stoufflet, Optimum Design Methods in Aerodynamics, AGARD-FDPVKI Special Course, April 25-29 (1994).

[2] A. Jameson, Aerodynamic Design Via Control Theory, Journal of Scientific Computing, 3:233-260 (1988).

[3] S. Ta'asan, G. Kuruvila and M. D. Salas, Aerodynamic Design and Optimization in One Shot, 30th Aerospace Sciences Meeting \& Exhibit, AIAA 92-0025, Jan. (1992).

[4] A. Iollo AND M. D. Salas, Contribution to the Optimal Shape Design of Two-Dimensional Internal Flows with Embedded Shocks, ICASE Report No. 95-20 (1995).

[5] A. Dervieux, J. Malé, N. Macro, J. Périaux, B. Stoufflet and H.Q. Chen, Some Recent Advances in Optimal Shape Design For Aeronautical Flows, Proceedings of "ECCOMAS, 2nd Computational Fluid Dynamics Conference", Sep. 5-8 (1994).

[6] P. D. Frank ANd G. R. Shubin, A comparison of optimization-based approaches for a model computational aerodynamics design problem, J. Comput. Phys. 98, 74 (1992).

[7] W. H. Jou, W. P. Huffman, D. P. Young, R. G. Melvin, M, B. Bieterman, C. L. Hilmes AND F. T. Johnson (The Boeing Company), Practical Considerations in Aerodynamic Design 
Optimization, AIAA 95-1730, 12th AIAA Computational Fluid Dynamics Conference, July 19-22 (1995).

[8] W. K. Anderson and V. Venkatakrishnan, Aerodynamic Design Optimization on Unstructured Grids with a Continuous Adjoint Formulation, AIAA 97-0643, 35'th Aerospace Sciences Meeting \& Exhibit, (1997).

[9] A. Jameson, N. A. Pierce, And L. Martinelli, Optimum Aerodynamic Design using the NavierStokes Equations, AIAA 97-0101, 35'th Aerospace Sciences Meeting \& Exhibit, (1997).

[10] M. B. Giles AND N. A. PIERCE, Adjoint Equations in CFD: duality, boundary conditions and solution behavior, AIAA 97-1850, (1997).

[11] R. M. LEwIS, A nonlinear programming perspective on sensitivity calculations for systems governed by state equations, ICASE Report No. 97-12, (1997).

[12] R. Dautray AND J-L Lions, Mathematical Analysis and Numerical Methods for Science and Technology, Vol. 2, Springer-Verlag, (1988).

[13] R. Courant and K. O. Friedrichs, Supersonic Flow and Shock Waves, Interscience Publishers, Inc., New York., p. 126 (1948).

[14] B. I. Soemarwoto, Multi-Point Aerodynamic Design by Optimization, Ph.D. thesis, Delft University of Technology, the Netherlands (1996).

Appendix A. Definition of the Euler Jacobian Matrices.

$$
\begin{aligned}
& A=\left(\begin{array}{cccc}
0 & 1 & 0 & 0 \\
-u^{2}+\frac{\gamma-1}{2}\left(u^{2}+v^{2}\right) & (3-\gamma) u & -(\gamma-1) v & \gamma-1 \\
-u v & v & u & 0 \\
-u\left(\gamma E-(\gamma-1)\left(u^{2}+v^{2}\right)\right) & \gamma E-\frac{\gamma-1}{2}\left(3 u^{2}+v^{2}\right) & -(\gamma-1) u v & \gamma u
\end{array}\right) \\
& B=\left(\begin{array}{cccc}
0 & 0 & 1 & 0 \\
-u v & v & u & 0 \\
-v^{2}+\frac{\gamma-1}{2}\left(u^{2}+v^{2}\right) & -(\gamma-1) u & (3-\gamma) v & \gamma-1 \\
-v\left(\gamma E-(\gamma-1)\left(u^{2}+v^{2}\right)\right) & -(\gamma-1) u v & \gamma E-\frac{\gamma-1}{2}\left(u^{2}+3 v^{2}\right) & \gamma v
\end{array}\right) .
\end{aligned}
$$

Appendix B. Identities of Polar Coordinates.

$$
\begin{gathered}
\operatorname{grad}(f)=\left(\frac{\partial f}{\partial r}, \frac{1}{r} \frac{\partial f}{\partial \theta}\right) \\
\operatorname{div}(\vec{u})=\frac{\partial u_{r}}{\partial r}+\frac{1}{r} \frac{\partial u_{t}}{\partial \theta}+\frac{u_{r}}{r} \\
\operatorname{grad}(\vec{u})=\left(\begin{array}{ll}
\frac{\partial u_{r}}{\partial r} & \frac{1}{r} \frac{\partial u_{r}}{\partial \theta}-\frac{u_{t}}{r} \\
\frac{\partial u_{t}}{\partial r} & \frac{1}{r} \frac{\partial u_{t}}{\partial \theta}+\frac{u_{r}}{r}
\end{array}\right) .
\end{gathered}
$$

If $A=A_{i j}$ is a tensor then

$$
\operatorname{div} A=\left(\begin{array}{l}
\frac{\partial A_{11}}{\partial r}+\frac{A_{11}}{r}+\frac{1}{r} \frac{\partial A_{12}}{\partial \theta}-\frac{A_{22}}{r} \\
\frac{\partial A_{21}}{\partial r}+\frac{A_{21}}{r}+\frac{1}{r} \frac{\partial A_{22}}{\partial \theta}+\frac{A_{12}}{r}
\end{array}\right) .
$$




\section{Appendix C. Demonstration of the Adjoint Derivation on a Complex Cost Functional Governed by the Compressible Navier-Stokes Equations.}

Let us take, for example, the following cost functional which is incomplete

$$
F=\int_{\Gamma}\left(\rho-\rho^{*}\right)^{2} d \sigma+\int_{\Gamma}\left(\frac{\partial p}{\partial n}-f^{*}\right)^{2} d \sigma
$$

The Lagrangian is given by

$(\mathrm{C} .2) \mathcal{L}=\int_{\Gamma}\left(\rho-\rho^{*}\right)^{2} d \sigma+\int_{\Gamma}\left(\frac{\partial p}{\partial n}-f^{*}\right)^{2} d \sigma+\int_{\Omega} \vec{\Lambda}^{T} \cdot(\nabla \cdot \vec{F}) d \Omega+\int_{\Gamma} \zeta \frac{\partial T}{\partial n} \sigma+\int_{\Gamma} \vec{\vartheta} \cdot \vec{u} \sigma+$ far-field terms.

The variation of the Lagrangian with respect to a change in the shape, $\bar{\alpha}$, is given by

$$
\begin{gathered}
\tilde{\mathcal{L}}=\text { interior terms }+\int_{\Gamma} 2 \tilde{\rho}\left(\rho-\rho^{*}\right) d \sigma+\int_{\Gamma} 2 \frac{\partial \tilde{\rho}}{\partial n}\left(\frac{\partial p}{\partial n}-f^{*}\right) d \sigma+ \\
\int_{\partial \Omega}\left[-\tilde{T} k \frac{\partial \lambda_{4}}{\partial r}+\frac{\partial \tilde{T}}{\partial r} k \lambda_{4}+\frac{\partial \tilde{u}_{r}}{\partial r} \frac{4}{3} \mu \lambda_{r}+\frac{\partial \tilde{u}_{t}}{\partial r} \mu \lambda_{t}+\tilde{p} \lambda_{r}\right] d \sigma
\end{gathered}
$$

For the cost functional (C.1) we need only the first two terms in Eq.(4.15) and therefore we choose $\eta_{3}=$ $\eta_{4}=0$ :

(C.4) $\tilde{\mathcal{L}}_{A B E}=\int_{\Gamma}\left[\eta_{1}\left(\frac{\partial \tilde{u}_{r}}{\partial r}\right)+\eta_{2}\left(\frac{\partial \tilde{p}}{\partial r}-\mu_{2} \tilde{\rho} \frac{1}{\rho^{2}} \frac{\partial \rho}{\partial t} \frac{\partial u_{t}}{\partial r}+\mu_{2} \frac{1}{\rho} \frac{\partial \tilde{\rho}}{\partial t} \frac{\partial u_{t}}{\partial r}+\mu_{2} \frac{1}{\rho} \frac{\partial \rho}{\partial t} \frac{\partial \tilde{u}_{t}}{\partial r}-2 \mu \frac{\partial^{2} \tilde{u}_{r}}{\partial r^{2}}-\mu \frac{\partial^{2} \tilde{u}_{t}}{\partial r \partial t}\right)\right.$.

The term $\frac{\partial^{2} \tilde{u}_{r}}{\partial r^{2}}$ doesn't cancel with any other term in the Lagrangian and, therefore, we add another ABE by taking the radial derivative of the continuity equation,

$$
\frac{\partial^{2}}{\partial r^{2}}\left(\rho u_{r}\right)+\frac{\partial^{2}}{\partial r \partial t}\left(\rho u_{t}\right)=0
$$

and restricting it to the boundary:

$$
\lim _{\vec{x} \rightarrow \Gamma} \frac{\partial}{\partial r} \operatorname{div}(\rho \vec{u})=\rho \frac{\partial^{2} u_{r}}{\partial r^{2}}+\frac{\partial \rho}{\partial t} \frac{\partial u_{t}}{\partial r}+\rho \frac{\partial^{2} u_{t}}{\partial r \partial t}=0 .
$$

We can further add to the variation of the Lagrangian (Eq.(C.3) added with Eq.(C.4)) the variation of the residuals of (C.6) with a Lagrange multiplier $\eta_{5}$ :

$$
\tilde{\mathcal{L}}_{n e w}=\tilde{\mathcal{L}}+\tilde{\mathcal{L}}_{A B E}+\int_{\Gamma} \eta_{5}\left(\tilde{\rho} \frac{\partial^{2} u_{r}}{\partial r^{2}}+\rho \frac{\partial^{2} \tilde{u}_{r}}{\partial r^{2}}+\frac{\partial \tilde{\rho}}{\partial t} \frac{\partial u_{t}}{\partial r}+\frac{\partial \rho}{\partial t} \frac{\partial \tilde{u}_{t}}{\partial r}+\tilde{\rho} \frac{\partial^{2} u_{t}}{\partial r \partial t}+\rho \frac{\partial^{2} \tilde{u}_{t}}{\partial r \partial t}\right) d \sigma
$$

The resulting adjoint equations on the boundary are given by ${ }^{4}$ (using $\tilde{T}=\frac{1}{\gamma-1}\left(-\frac{1}{\rho^{2}} \tilde{\rho}+\frac{1}{\rho} \tilde{p}\right)$ )

$$
\begin{array}{cc}
\frac{\partial \tilde{u}_{r}}{\partial r}(\Gamma): & \eta_{1}+\frac{4}{3} \mu \lambda_{r}=0 \\
\frac{\partial^{2} \tilde{u}_{r}}{\partial r^{2}}(\Gamma): & -2 \mu \eta_{2}+\eta_{5} \rho=0 \\
\frac{\partial \tilde{p}}{\partial r}(\Gamma): & \eta_{2}-2\left(-\frac{\partial p}{\partial r}-f^{*}\right)=0 \\
\tilde{p}(\Gamma): & \lambda_{r}-\frac{k}{\gamma-1} \frac{1}{\rho} \frac{\partial \lambda_{4}}{\partial r}=0 \\
\frac{\partial \tilde{u}_{t}}{\partial r}(\Gamma): & \mu \lambda_{t}+\eta_{2} \mu_{2} \frac{1}{\rho} \frac{\partial \rho}{\partial t}+\mu \frac{\partial \eta_{2}}{\partial t}-\frac{\rho \partial \eta_{5}}{\partial t}=0 \\
\tilde{\rho}(\Gamma): & 2\left(\rho-\rho^{*}\right)+\frac{k}{\gamma-1} \frac{1}{\rho^{2}} \frac{\partial \lambda_{4}}{\partial r}-\eta_{2} \mu_{2} \frac{1}{\rho^{2}} \frac{\partial \rho}{\partial t} \frac{\partial u_{t}}{\partial r}-\mu_{2} \frac{\partial}{\partial t}\left(\eta_{2} \frac{1}{\rho} \frac{\partial u_{t}}{\partial r}\right)+\eta_{5} \frac{\partial^{2} u_{r}}{\partial r^{2}}-\frac{\partial}{\partial t}\left(\eta_{5} \frac{\partial u_{t}}{\partial r}\right)+\eta_{5} \frac{\partial^{2} u_{t}}{\partial r \partial t}=\mathbf{0 .}
\end{array}
$$

The above system can be solved by first solving for $\eta_{1}, \eta_{2}$ and $\eta_{5}$ from the first three equations and then substituting the result in the last three equations, which are the desired adjoint boundary conditions.

\footnotetext{
${ }^{4}$ we assume that the terms defined on the endpoints of $\Gamma$ which result from the integration by parts are equal to zero.
} 


\begin{tabular}{|c|c|c|c|}
\hline \multicolumn{3}{|c|}{ REPORT DOCUMENTATION PAGE } & $\begin{array}{l}\text { Form Approved } \\
\text { OMB No. 0704-0188 }\end{array}$ \\
\hline \multicolumn{4}{|c|}{ 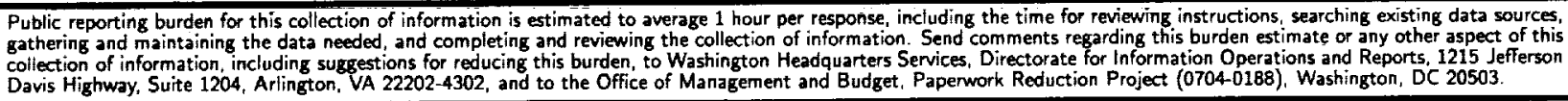 } \\
\hline 1. AGENCY USE ONLY(Leave blank) & $\begin{array}{l}\text { 2. REPORT DATE } \\
\text { December } 1997\end{array}$ & \multicolumn{2}{|c|}{$\begin{array}{l}\text { 3. REPORT TYPE AND DATES COVERED } \\
\text { Contractor Report }\end{array}$} \\
\hline \multicolumn{3}{|c|}{$\begin{array}{l}\text { 4. TITLE AND SUBTITLE } \\
\text { Admitting the Inadmissible: Adjoint Formulation for Incomplete Cost } \\
\text { Functionals in Aerodynamic Optimization }\end{array}$} & \multirow[t]{2}{*}{$\begin{array}{l}\text { 5. FUNDING NUMBERS } \\
\text { C NAS1-97046 } \\
\text { WU } 505-90-52-01\end{array}$} \\
\hline \multicolumn{3}{|l|}{$\begin{array}{l}\text { 6. AUTHOR(S) } \\
\text { Eyal Arian } \\
\text { Manuel D. Salas }\end{array}$} & \\
\hline \multicolumn{3}{|c|}{$\begin{array}{l}\text { 7. PERFORMING ORGANIZATION NAME(S) AND ADDRESS(ES) } \\
\text { Institute for Computer Applications in Science and Engineering } \\
\text { Mail Stop 403, NASA Langley Research Center } \\
\text { Hampton, VA 23681-0001 }\end{array}$} & $\begin{array}{l}\text { 8. PERFORMING ORGANIZATION } \\
\text { REPORT NUMBER } \\
\text { ICASE Report No. } 97-69\end{array}$ \\
\hline \multicolumn{2}{|c|}{$\begin{array}{l}\text { 9. SPONSORING/MONITORING AGENCY NAME(S) AND ADDRESS(E) } \\
\text { National Aeronautics and Space Administration } \\
\text { Langley Research Center } \\
\text { Hampton, VA 23681-2199 }\end{array}$} & $\begin{array}{l}\text { 10. SPONS } \\
\text { AGENC } \\
\text { NASA } \\
\text { ICASE }\end{array}$ & $\begin{array}{l}\text { 10. SPONSORING/MONITORING } \\
\text { AGENCY REPORT NUMBER } \\
\text { NASA/CR-97-206269 } \\
\text { ICASE Report No. } 97-69\end{array}$ \\
\hline \multicolumn{4}{|c|}{$\begin{array}{l}\text { 11. SUPPLEMENTARY NOTES } \\
\text { Langley Technical Monitor: Dennis M. Bushnell } \\
\text { Final Report } \\
\text { To be submitted to AIAA Journal }\end{array}$} \\
\hline \multicolumn{2}{|c|}{$\begin{array}{l}\text { 12a. DISTRIBUTION/AVAILABILITY STATEMENT } \\
\text { Unclassified-Unlimited } \\
\text { Subject Category } 64 \\
\text { Distribution: Nonstandard } \\
\text { Availability: NASA-CASI (301)621-0390 }\end{array}$} & & 12b. DISTRIBUTION CODE \\
\hline \multicolumn{4}{|c|}{$\begin{array}{l}\text { 13. ABSTRACT (Maximum } 200 \text { words) } \\
\text { We derive the adjoint equations for problems in aerodynamic optimization which are improperly considered as } \\
\text { "inadmissible". For example, a cost functional which depends on the density, rather than on the pressure, is } \\
\text { considered "inadmissible" for an optimization problem governed by the Euler equations. We show that for such } \\
\text { problems additional terms should be included in the Lagrangian functional when deriving the adjoint equations. } \\
\text { These terms are obtained from the restriction of the interior PDE to the control surface. Demonstrations of the } \\
\text { explicit derivation of the adjoint equations for "inadmissible" cost functionals are given for the potential, Euler, and } \\
\text { Navier-Stokes equations. }\end{array}$} \\
\hline \multirow{2}{*}{\multicolumn{3}{|c|}{$\begin{array}{l}\text { 14. SUBJECT TERMS } \\
\text { adjoint, aerodynamic optimization, Euler, Navier-Stokes, optimal shape }\end{array}$}} & $\begin{array}{l}\text { 15. NUMBER OF PAGES } \\
18\end{array}$ \\
\hline & & & $\begin{array}{c}\text { 16. PRICE CODE } \\
\mathrm{A} 03 \\
\end{array}$ \\
\hline $\begin{array}{l}\text { 17. SECURITY CLASSIFICATION } \\
\text { OF REPORT } \\
\text { Unclassified }\end{array}$ & $\begin{array}{l}\text { 18. SECURITY CLASSIFICATION } \\
\text { OF THIS PAGE } \\
\text { Unclassified }\end{array}$ & $\begin{array}{l}\text { 19. SECURITY CLASSIFICATION } \\
\text { OF ABSTRACT }\end{array}$ & $\begin{array}{l}\text { 20. LIMITATION } \\
\text { OF ABSTRACT }\end{array}$ \\
\hline NSN 7540-01-280-5500 & & & $\begin{array}{l}\text { Standard Form 298(Rev. 2-89) } \\
\text { Prescribed by ANSI Std. 239-18 } \\
\text { 298-102 }\end{array}$ \\
\hline
\end{tabular}

